\title{
La enseñanza de la geometría analítica en la educación media
}

\section{The teaching of analytic geometry in secondary school}

\begin{abstract}
Armando Aroca Araújo ${ }^{1}$
${ }^{1}$ Candidato a Doctor, del Doctorado Interinstitucional de Educación de la Universidad Distrital Francisco José de Caldas, énfasis en Educación Matemática, DIE-UDFJC, Profesor Asociado de la Universidad del Atlántico, e-mail: armandoaroca@mail.uniatlantico.edu.co, Dhttp://orcid.org/0000-0003-2786-4848
\end{abstract}

Cómo citar: Aroca Araújo, A. 2019. La enseñanza de la geometría analítica en la educación media. Rev. U.D.C.A Act. \& Div. Cient. 22(1):e1222. https://doi.org/10.31910/rudca.v22.n1.2019.1222

Artículo de acceso abierto publicado por Revista U.D.C.A Actualidad \& Divulgación Científica, bajo una licencia Creative Commons CC BY-NC 4.0

Recibido: Agosto 17 de 2018

Aceptado: Mayo 17 de 2019

\section{RESUMEN}

El problema de investigación fue comprender el porqué de la enseñanza de un conjunto de temas que caracterizaron un curso de Geometría Analítica de grado 10, durante un periodo determinado. El objetivo de esta investigación fue, entonces, describir y analizar el porqué de la selección de dicho conjunto de temas caracterizaron el estudio de caso. Para lograr este objetivo fue necesario hacer una investigación etnográfica en un aula de clases, de una institución educativa pública, ubicada al norte de Colombia. La metodología empleada respondió a dos momentos: 1) Una observación no participante y su respectivo registro audiovisual y transcripción de 14 sesiones de clases y la revisión de cuadernos y 2) El análisis de la información obtenida en el anterior momento, conllevó al empleo de una metodología de revisiones bibliográficas y documentales, sobre el desarrollo curricular en el contexto internacional y nacional de la enseñanza de la Geometría Analítica, en la educación media, para relacionar el análisis del porqué de la enseñanza del conjunto de temas con los aspectos que emergieron de dicha revisión. Al final, se aporta a la discusión sobre lo que podría ser el curso de Geometría Analítica del grado 10.

Palabras clave: Geometría Analítica; enseñanza; contenidos; currículo; perspectivas de cambio.

\section{ABSTRACT}

The research problem was to understand the why of the teaching of a set of topics that characterized a grade 10 Analytical Geometry course during a given period. The objective of this investigation was then to describe and analyze why the selection of said set of topics characterized the case study. To achieve this goal it was necessary to do ethnographic research in a classroom of a public educational institution located in northern Colombia. The methodology used responded to two moments: 1) A non-participant observation and its respective audiovisual record and transcript of 14 class sessions and the revision of notebooks and 2) The analysis of the information obtained in the previous moment led to the use of a methodology of bibliographic and documentary reviews on curricular development in the international and national context of the teaching of Analytical Geometry in secondary education to relate the analysis of why of the teaching of the set of topics with the aspects that emerged from said review. At the end it is contributed to the discussion about what could be the course of Analytical Geometry of grade 10.

Keywords: Analytic geometry; teaching; content; curriculum; prospects for change. 


\section{INTRODUCCIÓN}

Como lo atestiguan Jones \& Tzekaki (2016), hay muy poca investigación sobre los aspectos relacionados con la enseñanza de la Geometría Analítica, tanto en su didáctica como en la historia de su didáctica, en los distintos niveles de la educación. Un sondeo informal y su propia experiencia pueden convencer al lector de que, en todas las ciudades de su país y en todos los colegios de su ciudad, se enseñan, en algún grado de la educación media, al menos, cinco temas centrales de la Geometría Analítica de Descartes: las coordenadas cartesianas de un punto (x,y), la distancia entre dos puntos, la ecuación de la recta, la ecuación cuadrática y las secciones cónicas. Lo anterior es motivo para investigar qué sucede hoy día con la enseñanza de la Geometría Analítica en la educación media, en Colombia. En esta investigación, se plantea parte de ese reto, a partir de la búsqueda documental y de un estudio de caso.

Este escrito abarca varias partes; la primera presenta una revisión de literatura sobre el desarrollo curricular de la Geometría Analítica en el contexto internacional, que empieza desde finales del siglo XIX y la segunda, de carácter nacional con énfasis en la construcción de referentes legislativos y curriculares, liderados por el Ministerio de Educación Nacional. Esta revisión, se hizo para construir una aproximación al análisis de la selección de un conjunto de temas que se enseñaron en un curso de Geometría Analítica de grado 10, que se observó durante el periodo 2017-2. Hacer dicha aproximación es lo que se constituyó como problema de investigación, pues se desconocían razones del porqué de los temas, su orden y la duración de la enseñanza en las clases. En otra parte del artículo, se presentan los materiales y los métodos empleados para el estudio de caso y se omite la metodología empleada para la revisión bibliográfica y gestión documental; no obstante, se tuvieron en cuenta las recomendaciones de Gómez-Luna et al. (2014). Luego, se procede a poner en relación los aspectos emergentes en la revisión de literatura, con las descripciones de las 14 sesiones del estudio de caso. Por último, se presentan las limitaciones de la investigación, algunas conclusiones y recomendaciones, sobre lo que podría ser el curso de Geometría Analítica del grado 10, de la educación media colombiana.

Una revisión de literatura de corte histórico. El contexto internacional. La revisión parte desde finales del siglo XIX. La razón de ello es que, desde esta época, con base en el Programa de Erlangen de Klein, de 1872, se establecía el cese de las disputas entre las geometrías sintética y analítica, pues, bajo este punto de vista, una «geometría» vendría a ser el conjunto de propiedades invariantes a partir de las transformaciones del grupo correspondiente (Bourbaki, 1969). A finales del siglo XIX, la matemática alemana tenía una tendencia a unificar "todo el saber matemático sobre una base única, abstracta, que se apartaba de la "intuición”" (Cocho, 1985). Esta tendencia todavía domina las matemáticas más avanzadas, como la geometría algebraica y la geometría, como álgebra lineal, sin figuras que propuso Bourbaki y sigue vigente en Francia y otros países; sin embargo, la separación de la geometría y la intuición hay que analizarlo más; por ejemplo, según Jones \& Tzekaki (2016), la geometría comprende aquellas ramas de las matemáticas que explotan la intuición visual, la más dominante de nuestros sentidos.

La UNESCO (1986) publicó una compilación de varias investigaciones sobre la enseñanza de la geometría en varias regiones del mundo. Aquí, Lluis (1986) hizo un análisis de la enseñanza de la geometría en América Latina. Este autor considera que la principal característica de su enseñanza es la duda sobre lo que se debe enseñar. Concluye que, desde principios del siglo XX hasta la década del 60 , se enseñaron los mismos temas de geometría, tanto en el nivel primario como en la secundaria. Este reporte manifiesta que, para 1960, la enseñanza de la geometría en secundaria alcanzó su más bajo nivel en la calidad de la enseñanza. Por ello, en esta década, se dieron cambios, lo cual, generaría un problema que se hizo notar varios años después (Howson, 1973): durante la década de 1960, los recursos pedagógicos, especialmente textos escolares, fueron traducidos y transferidos entre los países, pero con insuficiente atención prestada a las diferencias sociales, educativas y culturales. Desde la década del 60, el desarrollo histórico de la enseñanza de la Geometría ha tenido la incertidumbre de qué, cómo y para qué enseñar Geometría en América Latina (Unesco, 1986).

Por su parte, Bishop (2005) plantea que existen ciertos obstáculos para aprender acerca del espacio en las aulas escolares, entre ellos, el énfasis en la aritmética y la poca conexión de las actividades curriculares de enseñanza con el mundo espacial fuera del aula. Sobre lo anterior, Bishop complementa diciendo que, desde el punto de vista cultural, debemos reconocer que, para muchos alumnos en el mundo, las ideas geométricas que se les está enseñando en la escuela son consecuencia de una forma de ver el mundo espacial, que es completamente distinta de la forma como se ve esa realidad en su "cultura familiar". Puede haber niños pertenecientes a familias que se han trasladado recientemente a otro país, o que pueden pertenecer a una población, cuya cultura queda eclipsada por un grupo cultural más dominante, dentro de aquel país. El trabajo realizado por Harris (1984) con aborígenes australianos y el de Pinxten et al. (1983), con los indios navajos en los Estados Unidos de América, presentan muy claramente algunos de estos problemas. Esta postura de Bishop, se complementa con diversos trabajos de Gerdes (1996; 1999; 2014) y otras investigaciones que él mostró sobre la relación entre geometría y contexto, entre la cestería, el papel de la mujer y la geometría; el análisis de los entrecruces que se hacen con cuerdas, los diseños que se hacen en la arena, entre otros. El conjunto de estas investigaciones advierte sobre el problema de enseñar una geometría impuesta de otros contextos y dejar de lado las representaciones sobre espacio y tiempo y sobre los fenómenos de la cotidianidad y del mundo de los estudiantes y los profesores.

En la década del 70, con el prólogo del libro "Álgebra lineal y geometría elemental” (Dieudonné, 1964), el autor rechazó la separación de lo que denomina pseudociencias (e.g., la geometría sintética, la geometría analítica y la trigonometría, entre otras), en la enseñanza tradicional. Algunos autores planteaban que era necesario discutir el tipo de geometría que se debía enseñar en la educación secundaria, entre ellos, matemáticos franceses, como 
Choquet, Dieudonné, Godement y Artin (Austriaco), Freudenthal (neerlandés-alemán), Santaló (español), en la década de los sesenta (Piaget et al. 1978).

En el caso concreto de la geometría en la secundaria, Santaló (1980), se refirió a la urgencia de establecer nuevos currículos, considerando las capacidades de aprendizaje de los estudiantes. Esta consideración, se había hecho desde los años 60 , con los programas curriculares elaborados con los objetivos de Bloom, los cuales, se graduaban para cada edad, en casi todos los países latinoamericanos. Gascón (2002) propuso, a través de cierto tipo de problemas, una forma de conectar el enfoque sintético con el analítico, a fin de poner de manifiesto su complementariedad; afirma que aún hay una discusión abierta sobre el tipo de geometría por enseñar en la educación secundaria.

Lo anterior, se enlaza con la revisión documental que hicieron Jones \& Tzekaki (2016), sobre los trabajos que se presentaron en las conferencias anuales de PME (The International Group for the Psychology of Mathematics Education), durante el período 2005-2015. De la extensa revisión de artículos que hicieron, se pudo verificar que la investigación en Geometría Analítica/Coordenadas es limitada, como también en geometría vectorial.

Los aspectos que emergieron en la revisión de literatura de corte histórico internacional son los siguientes:

1. Disputa entre geometría sintética y geometría analítica.

2. Complementariedad entre los enfoques sintético y analítico.

3. Distanciamiento de la intuición.

4. Dudas sobre qué enseñar.

5. Indiferencia de textos escolares a diferencias sociales, educativas y culturales.

6. Desconexión de las actividades curriculares con el mundo espacial fuera del aula.

7. Novedad de currículos basados en las capacidades de los estudiantes

En el análisis del estudio de casos, que se presentará más adelante, estos aspectos serán utilizados para identificar distanciamientos o acercamientos de dicho caso.

La construcción de referentes legislativos y curriculares. Para principios del siglo XX, el Ministerio de Instrucción Pública, promulgó el Decreto 491 de 1904 y en su artículo 115, referido a la enseñanza en las Escuelas Normales, en su Inciso 15 y en el artículo 120, sobre la instrucción secundaria clásica, comprenderá todas las enseñanzas de Letras y Filosofía, para el efecto de cursar en las Facultades universitarias, mediante el diploma de Bachiller en Filosofía y Letras, consideró que se debía contemplar la enseñanza de la Geometría plana y del espacio y, en el caso de las Escuelas Normales de varones, solamente se debían realizar ejercicios prácticos de Agrimensura. No fue posible encontrar los contenidos de estos cursos. De Geometría Analítica, se hablaba solo en las carreras de Ingeniería y de Matemáticas, como lo establece el Capítulo III. Los contenidos de este curso siguen siendo, por lo general, los que hoy día conocemos. Esto último ratificado por
Torres-Sánchez \& Salazar-Hurtado (2002), en la reconstrucción que hicieron de la historia de la ingeniería y de la educación en Colombia.

A la primera mitad del siglo XX, le da la mano el inicio de reformas educativas para el sistema escolar de Colombia. Desde 1951 hasta el 2017, han existido reformas que han tenido relación directa o indirectamente con la enseñanza de la Geometría Analítica, en la educación media. Los decretos o reformas que hacen parte de este proceso histórico, se describen así: Decreto 0075 de 1951 del MinEducación, se adopta el Plan de Estudios para la enseñanza secundaria y se dictan otras disposiciones y aparece, sin contenidos ni objetivos específicos, para el quinto de bachillerato el curso de Geometría, correspondiente al actual décimo grado. Con el Decreto 2550 de 1951 del MinEducación, aparece el curso de Geometría y Trigonometría para el año quinto de bachillerato, dicho decreto solo promulga los programas específicos anunciados en el Decreto 0075; con el Decreto 45 de 1962 del MinEducación aparecen en quinto de bachillerato los cursos de Trigonometría y Elementos de Geometría Analítica; en este decreto, en sus consideraciones, se estableció: "Que el Gobierno Nacional, para mejorar la calidad de la educación media y atender a su mayor demanda, ha venido estudiando un plan fundamental mínimo de estudios, en consonancia con las modernas tendencias educativas y las necesidades del país". Con el Decreto 080 de 1974, conocido como la reforma de la educación media, se deroga el Decreto 45 de 1962 y se producen seis folletos cortos, en papel periódico, con los programas de cada grado de secundaria. El Decreto 1419 de 1978 del MinEducación hizo énfasis en las normas y las orientaciones básicas para la administración curricular en los niveles de educación preescolar, básica (primaria y secundaria), media vocacional e intermedia profesional; en el Decreto 1002 de 1984 del MinEducación, se establece el Plan de Estudios para la Educación Preescolar, Básica (Primaria y Secundaria) y Media Vocacional; en su capítulo III establece la organización y la distribución de 30 horas para la educación media vocacional.

Ninguno de los decretos anteriores especificó contenidos para los cursos establecidos; sin embargo, se procedió a consultar a tres profesores que enseñaron Geometría Analítica en grado 10, en la década del 80, del 90 y en el 2017. No fue posible encontrar un profesor que haya enseñado dicho curso en la década del 70 . Se les pidió a los profesores que escribieran cuáles fueron los contenidos que enseñaron y que explicaran cómo habían obtenido la información; todos coincidieron que lo obtuvieron de un texto guía. El profesor que enseñó a principios de la década de los 80 planteó el siguiente programa: Antecedentes históricos; ecuación cartesiana de la circunferencia; circunferencia circunscrita a un triángulo; la parábola; parábola con vértice en $(\mathrm{h}, \mathrm{k})$; la elipse como lugar geométrico; definición y elementos; construcción de una elipse; principales propiedades de la elipse; ecuación cartesiana de una elipse de centro en el origen y cuyos ejes coinciden con los ejes coordenados; determinación de los principales elementos de una elipse, dada en la primera forma ordinaria; ecuación de una elipse de centro un punto cualquiera y ejes paralelos a los coordenados; determinación de los elementos de una elipse dada en su segunda forma ordinaria; definición de la hipérbola; hipérbola con centro en el origen; asíntotas de la hipérbola; excentricidad de la hipérbola; 
hipérbolas con eje focal paralelo a un eje cartesiano. Su fuente para la organización de los contenidos anteriores, se basó en su propia experiencia docente como en varios textos de la época, principalmente, el de Patiño (1977). A esta información, se le sumó un comentario del profesor que nos muestra el tiempo destinado para el curso de Geometría Analítica en grado 10: "Normalmente, lograba desarrollar las tres primeras cónicas, pues la Geometría Analítica se trabajaba en el último período académico, después de terminar la Trigonometría".

Un segundo profesor, quien enseñó a finales de la década del 90, planteó el siguiente programa: Distancia entre dos puntos en el plano cartesiano; punto medio de un segmento; la recta; las cónicas; la circunferencia y sus elementos; puntos de corte entre una circunferencia y una recta; problemas aplicados a la circunferencia; la parábola y sus elementos; puntos de corte entre una parábola y una recta; problemas aplicados a la parábola; la elipse y sus elementos; puntos de corte entre la elipse y una recta; problemas aplicados a la elipse; la hipérbola y sus elementos; puntos de corte entre una hipérbola y una recta; problemas aplicados a la hipérbola. Los contenidos anteriores no fueron organizados por el profesor, sino que le fueron dados por la Fundación universitaria que lo había contratado; sin embargo, el profesor manifestó que tenía la libertad de consultar libros o textos de matemáticas, entre ellos, libros que eran usados a nivel universitario, como Lehmann (1989), Leithold (1998), entre otros, pero también usó otros textos para la educación media. Las diferencias y las coincidencias entre ambos cursos son notorios, al preguntarles por qué no habían enseñado ciertos temas y otros sí; se notó que la razón respondía a criterios personales.

En 2006 aparecen los Estándares Básicos de Competencias en Matemáticas. Dos de los seis estándares básicos de competencia con respecto al pensamiento espacial y sistemas geométricos, se establecen como: "Identifico características de localización de objetos geométricos en sistemas de representación cartesiana y otros (polares, cilíndricos y esféricos) y en particular de las curvas y figuras cónicas", la negrilla es nuestra; "Resuelvo problemas en los que se usen las propiedades geométricas de figuras cónicas por medio de transformaciones de las representaciones algebraicas de esas figuras" (Schmidt, 2006). Estos dos estándares son alusivos a los cuatro temas que se han mencionado desde el principio del artículo. En el 2015, se dan debates sobre los cuestionados Derechos Básicos de Aprendizaje para Matemáticas (DBA) y en el 2016 aparecen, formalmente, ante toda la comunidad académica (MinEducación, 2016). En el 2017, se presentan las Mallas de aprendizaje para todo el ciclo escolar, pero el Ministerio de Educación Nacional decide publicar solo los de la educación básica primaria. Estos dos últimos documentos aún hacen parte del debate académico.

$\mathrm{Al}$ retomar las experiencias de los profesores, un tercer profesor, quien enseñó en el 2017, planteó el siguiente programa: La línea recta y ejercicios; la ecuación de segundo grado y ejercicios; secciones cónicas; la circunferencia; ecuación canónica de la circunferencia con centro en (h,k); ecuación general de la circunferencia y ejercicios; problemas de aplicación; la parábola; ecuación canónica de la parábola con centro en (h,k); ecuación general de la parábola y ejercicios; problemas de aplicación; la elipse; ecuación canónica de la elipse con centro en $(\mathrm{h}, \mathrm{k})$; ecuación general de la elipse y ejercicios; problemas de aplicación; la hipérbola; ecuación canónica de la hipérbola con centro en $(\mathrm{h}, \mathrm{k})$; ecuación general de la hipérbola y ejercicios; problemas de aplicación. Su fuente fue el texto de Riaño (2017). Si se toma como referencia los programas desarrollados por los tres profesores, ellos tienen algunas coincidencias: ausencia del paso formal a $3 \mathrm{~d}$ en la representación del espacio con tres variables $\mathrm{x}, \mathrm{y}$, z. Cuando se les preguntó por los problemas de aplicación, los tres profesores tuvieron respuestas similares, como, por ejemplo: el barco que se acerca al muelle, el acantilado muy perpendicular al suelo, el vuelo del avión, la forma del túnel o de la antena, entre otros clásicos problemas que, hoy día, en ediciones nuevas de Matemáticas 10 , se siguen encontrando.

Se puede inferir que el curso de Geometría Analítica en grado $10^{\circ}$ de Colombia data explícitamente desde 1962, con el Decreto 45, con cursos de Trigonometría y elementos de Geometría Analítica, con antecedentes en la década del 50, mediante cursos de Geometría y Trigonometría. Los temas que se han enseñado durante los siglos XX y lo que corre del XXI mantienen una base común, como también la ausencia del paso a 3d y que los problemas de aplicación son similares. Si se pone en relación con los aspectos que emergieron en la literatura de corte histórico internacional, se abona que no hubo dudas sobre qué enseñar, pero se mantiene la indiferencia de textos escolares a diferencias sociales, educativas y culturales y la desconexión de las actividades curriculares con el mundo espacial fuera del aula.

Revisión de textos escolares editados en el periodo de la construcción de referentes legislativos y curriculares. Después de analizar algunos textos escolares de matemáticas, por ejemplo, Phillips (1948), Postigo (1964), Dolciani et al. (1972), y Riaño (2017), que fueron empleados por profesores de la educación media, en diversos momentos históricos, los contenidos de Geometría Analítica mantienen una base en común, que son: estudio de la línea recta, la ecuación general de segundo grado, las secciones cónicas representadas principalmente en el sistema cartesiano de $2 \mathrm{~d}$, lo que implica que existe una ausencia del paso formal a $3 \mathrm{~d}$ en la representación del espacio, con tres variables x, y, z. En estos libros, se notó que varios de los problemas que se denominan de aplicación, se empezaron a volver frecuentes con el paso de los años, solo se cambiaban los datos y las representaciones, como los del barco, el avión, la antena, entre otros. Con lo anterior, se podría plantear, que el aspecto de la Indiferencia de textos escolares a diferencias sociales, educativas y culturales, sigue siendo una constante en este tipo de recurso pedagógico del profesor. La repetición misma de los problemas de aplicación muestra su desconexión de las actividades curriculares con el mundo espacial fuera del aula. Nos hemos acostumbrado a ser víctimas de la comodidad que presenta el hecho de que los ejemplos o problemas de aplicación que nos presentan los textos escolares, ya están dados e, incluso, en algunos casos, resueltos. Bastaría que el profesor de matemáticas indague un poco en su propio contexto sociocultural, en el entorno próximo 
de su Institución Educativa, en los problemas tecnológicos actuales, para que contextualice, de una manera más llamativa, las situaciones didácticas en el aula de clases de la Geometría Analítica de grado 10.

\section{MATERIALES Y MÉTODOS}

Para Stake (1999), el objetivo primordial del estudio de un caso no es la comprensión de otros. La primera obligación es comprender este caso. Por ello, el estudio del caso no tiene como propósito la generalización sino la particularización de la enseñanza de la Geometría Analítica en grado 10. La Institución Educativa donde se recogió la información es de carácter público, mixta, con 35 estudiantes y ubicada en la ciudad de Barranquilla, Colombia.

Se tomó como referentes a Stake (1999) y Hernández et al. (2014), para la recolección y análisis de la información. El tipo de investigación, se caracterizó por ser de carácter etnográfico, que incluyó observación no participante y recolección de información, por medio del registro audiovisual de 14 clases, que comprendieron el semestre 2017-2, más sus respectivas transcripciones. En artefactos, como cuadernos, talleres y exámenes de estudiantes, también se recogió información, para el análisis de las clases desarrolladas en los dos periodos académicos, comprendidos en el 2017-1; esta información, se confrontó con los contenidos del texto Guía del estudiante de Riaño (2017). En el análisis de la información obtenida que representaron el 2017 fue necesario hacer una revisión de literatura de corte histórico y de los referentes curriculares y legislativos, para tener elementos de comprensión de la constitución del estudio de caso. Las clases observadas eran de enseñanza tradicional y, en ninguna, se empleó software geométrico.

\section{RESULTADOS Y DISCUSIÓN}

En el cuadro 1 se describen las 14 clases que se desarrollaron en el periodo 2017-2, en un curso de Geometría Analítica de la educación media colombiana y de lo que se enseñó en el periodo 2017-1. Según esta información, lo que realmente se enseñó fueron los temas sobre Ecuación de la recta, Ecuación de segundo grado y las Secciones Cónicas, excepto la hipérbola.

Al poner en relación los aspectos emergentes de la revisión de literatura de corte histórico del desarrollo internacional, el periodo de cambios, por lo menos teóricos, que se dieron por medio de referentes legislativos y curriculares desde la aparición del Ministerio de Educación Nacional, con la revisión documental de cuadernos, talleres y exámenes del periodo 2017-1 y la observación de las 14 clases del periodo 2017-2 de un curso de Geometría Analítica en grado 10, se podría establecer lo siguiente sobre dicho curso:

1. Se mantiene el distanciamiento de la intuición, pues al revisar las transcripciones de clases se encontraron varios momentos en que el profesor no tiene en cuenta procesos subjetivos, creativos y el empleo de artefactos que no estaban validados, como instrumentos geométricos. También soportamos esta relación por medio de Alsina et al. (1987), quienes consideran que uno de los modos de comprensión y de expresión del espacio geométrico, que se pueden considerar como fases del desarrollo del pensamiento, se realiza de forma directa, creativa y subjetiva de naturaleza visual y corresponde a lo que podríamos llamar intuición geométrica. Esta distancia de la intuición ubica, entonces, el estudio de caso con la advertencia que Cocho (1985) hacía sobre la matemática alemana de finales del siglo XIX y su tendencia de unificar todo el saber matemático sobre una base única, abstracta.

2. Desde el 2006, no hay dudas sobre qué enseñar. Hay un tipo de certeza sobre lo que se debe enseñar, pues existen los Lineamientos y Estándares Curriculares, Mallas, DBA y hasta un texto escolar: Guía del docente (Riaño, 2017), dado gratuitamente por el MEN. Esto implica que el profesor contaba con un recurso pedagógico, que no le generaría dudas sobre qué enseñar, contrario a lo sucedido en la década del 60.

3. Indiferencia de textos escolares a diferencias sociales, educativas y culturales, pues los textos Guía del docente y Guía del estudiante, siguen de espaldas a las diferencias sociales, educativas y culturales, tal como Howson (1977) había manifestado sobre la enseñanza de la Geometría Analítica, en la década del 60. Colombia es un país con una alta diversidad cultural y ello implica diferencias sociales y educativas, que los textos guías de matemáticas podrían, de forma estratégica incluir, más allá de hacer énfasis en aplicaciones tradicionales o clásicas, como la trayectoria de un avión, el diseño de engranajes de piñones, distancias de partes de un puente, diseño de arandelas.

4. Se mantiene la desconexión de las actividades curriculares con el mundo espacial fuera del aula, también advertida en la década del 60, con el agravante de que, hoy día, el desarrollo tecnológico demanda transformaciones curriculares, para comprender mejor nuevos sistemas de orientación, de dirección, de ubicación, en general, ese mundo espacial que hace parte de las experiencias de los estudiantes y los profesores. La advertencia que hizo Bishop (2005), sobre las distancias que hay entre las representaciones que se tienen del mundo espacial en la cotidianidad y la forma en que la interpreta la escuela, siguen hoy día vigentes, por lo menos, en el estudio de caso que se escogió. Son diversos los episodios evidenciados en las transcripciones de clases que así lo sustentan. Uno de ellos es un problema de aplicación de la circunferencia que le fue asignado a una estudiante para que expusiera en clase. Tanto el problema, el contexto y la experiencia que evoca la actividad, le son muy ajenos a los estudiantes.

5. Em: jlisto!/ dice/ este es un poco más complejo/ pero //y dice/ un servicio sismológico de Baja California/ detectó un sismo con origen en la ciudad de Mexicali/ en el origen es Mexicali/ y dice/ a cinco kilómetros este y tres kilómetros sur del centro de la ciudad/ con un radio de cuatro kilómetros a la redonda/ ¿Cuál es la ecuación de la circunferencia del área afectada? / / utilizando esta ecuación indica si afectó a la ciudad de Mexicali/// ee/ bueno/ recordando/ norte/ sur/ este/ y oeste/ entonces nos dice que está/ cinco kilómetros al este/ un / dos/ tres/ cuatro/ cinco / / cinco kilómetros al este y que está tres kilómetros sur/ tres kilómetros sur/ entonces/ este vendría siendo el punto. 
Cuadro 1. Descripción de 14 sesiones de clases de un curso de Geometría Analítica de grado 10 en Colombia.

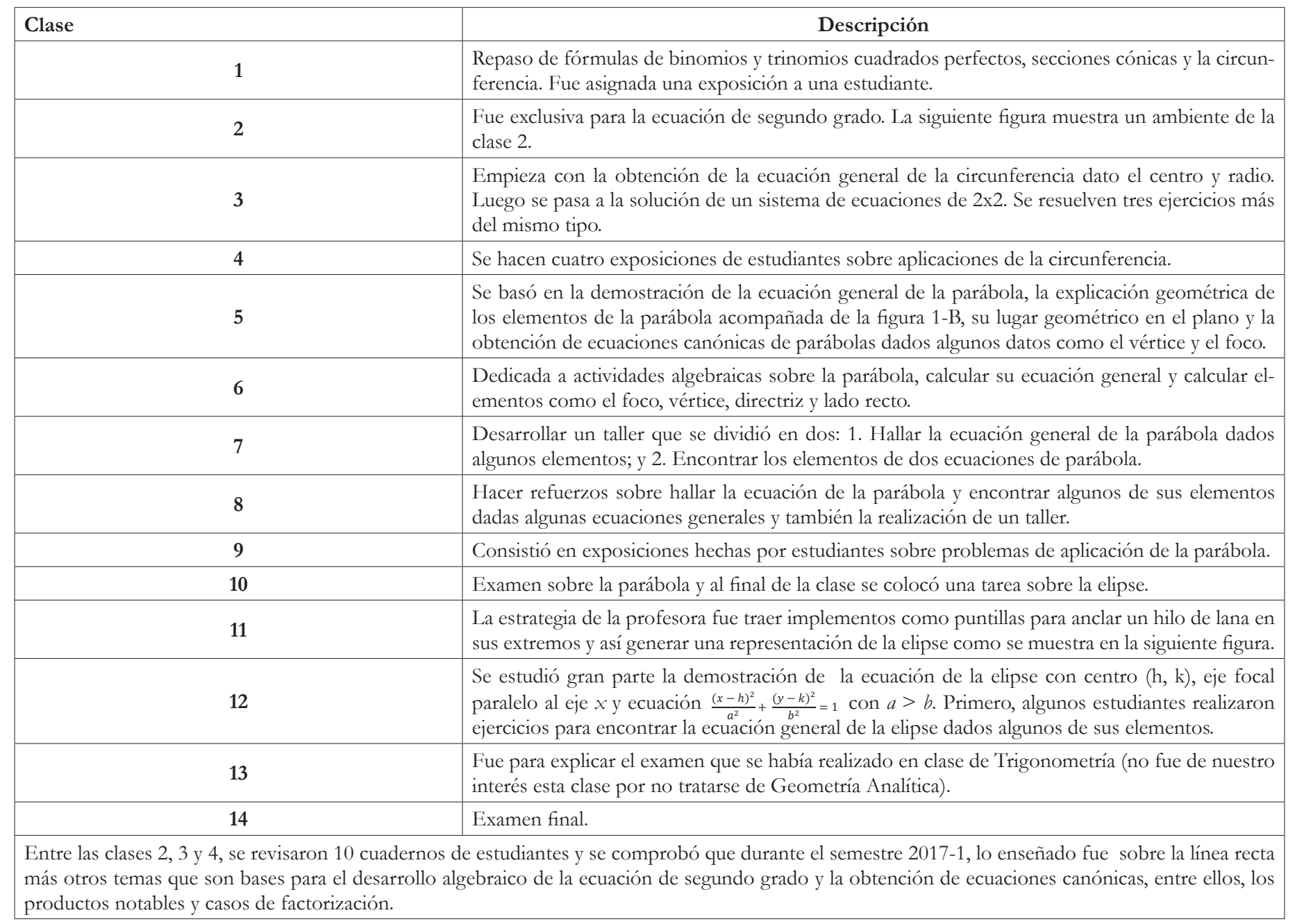

Del presente trabajo se concluye que las limitaciones de la investigación son las que se derivan del estudio de caso, donde se escogió un solo curso de Geometría Analítica. Por ello, solo hay elementos de este caso, que invitan a la reflexión sobre la organización temática del curso de Geometría Analítica de grado $10^{\circ}$ y su mayor conexión social con la vida de los profesores y de los estudiantes. Algunas investigaciones que podrían ayudar en la pretensión de cambio de este curso son resultados de investigación del Programa Etnomatemática, entre ellos, la diversidad cultural que hay para encontrar la ruta, puntos de referencias y orientación (Lewis, 1976); los tres niveles de espacio (Pinxten et al.1983); las relaciones íntimas entre las estaciones, las direcciones, la temperatura y el sol (Harris, 1984); el análisis sobre el desarrollo del pensamiento espacial basado en: 1) el marco de referencia y 2) las influencias del lenguaje, en cómo las personas piensan, memorizan y razonan sobre las relaciones espaciales y las direcciones (Levinson, 2003). De este último autor, se puede concluir que nos orientamos espacialmente en un contexto específico, según nuestro lenguaje, algo que también afirma Barton (2008) y Tenbrink \& Kuhn (2011) y las recientes investigaciones de Owens (2015) y Leal (2015), lo que pondría en entredicho el lenguaje empleado en los textos escolares de Geometría Analítica, que son pensados para un "público de lenguaje universal"; sin embargo, el debate sería: ¿se emplea un lenguaje "universal" que pueda ser interpretado y contextualizado en cualquier parte del planeta o se crean tantos lenguajes matemáticos para la Geometría Analítica como contextos existan?

El curso de Geometría Analítica en la educación media juega un papel importante en el desarrollo de representaciones espaciales del estudiante y de los profesores y en las conexiones con otros problemas matemáticos, como el razonamiento espacial, la visualización o el uso del lenguaje. El manejo de la representación transcultural del plano cartesiano y las ecuaciones algebraicas, que correspondan a lugares geométricos transculturalmente reconocidos, puede permitir una mejor comprensión del objeto matemático. Por ello, se plantea, a la luz de los cambios tecnológicos que hacen parte de la cotidianidad de las poblaciones en procesos de escolarización, una discusión para construir un currículo acorde con el mundo moderno y las diferencias sociales y culturales. 
El reto es que la Geometría Analítica en la educación media deje de aplanar la imaginación en $2 d$, que deje la hegemonía del plano cartesiano en $2 d$ y pasemos a su espacialización, incluyendo una tercera dimensión, el eje z. Para que lo analítico se espacialice es necesario incluir dicho eje, "avalada" por los Lineamientos y Estándares Curriculares, teniendo, como consecuencia, diversas situaciones didácticas, como el estudio de fenómenos en $3 d$ y la misma formación profesional y didáctica del maestro.

Conflicto de intereses: El manuscrito fue preparado y revisado por el autor, quien declara ser el único autor y que no existe conflicto de intereses, que ponga en riesgo la validez de los resultados presentados. Este artículo es un resultado de la tesis doctoral titulada "Formas de operación y de expresión de los modelos mentales cronotópicos de un profesor y sus estudiantes cuando desarrollan actividades que vinculan el uso de coordenadas en el plano y en el espacio en clase de geometría analítica de grado $10^{\circ}$ ' del DIEUDFJC.

\section{REFERENCIAS}

1. ALSINA, C.; BURGUÉS, C.; FORTUNY, J.Ma. 1987. Invitación a la Didáctica de la Geometría. Síntesis (Madrid).144p.

2. BARTON, B. 2008. The Language of Mathematics. Springer (Australia). 186p.

3. BISHOP, A. 2005. Aproximación Sociocultural a la Educación Matemática. Programa Editorial Universidad del Valle. (Cali). 199p.

4. BOURBAKI, N. 1969. Formas cuadráticas. Geometría elemental. En: Hernández, J. (ed.). Elementos de historia de las matemáticas. Alianza Editorial (España). p.173-191.

5. COCHO, F. 1985. El Programa de Erlangen. Felix Klein. Revista del Seminario de Enseñanza y Titulación. (México). 2(49):1-3.

6. DIEUDONNÉ, J. 1964. Prólogo del libro "Álgebra lineal y geometría elemental”. En: Piaget, J.; Choquet, G.; Dieudonné, J.; Thom, R. La enseñanza de las matemáticas modernas, Alianza Editorial, Madrid.

7. DOLCIANI, M.; BERMAN, S.; FREILICH, J. 1972. Álgebra moderna. Publicaciones cultural S.A. (México). 566p.

8. GASCÓN, J. 2002. Geometría sintética en la ESO y analítica en el Bachillerato. ¿Dos mundos completamente separados? SUMA, Revista sobre la enseñanza y aprendizaje de las Matemáticas. (España). 39:13-25.

9. GERDES, P. 1996. Women, Culture and Geometry in Southern Africa. Lulu. (Mozambique). 276p.
10. GERDES, P. 1999. Geometry from Africa: Mathematical and Educational Explorations. The mathematical Association of America. (U.S.). 210p.

11. GERDES, P. 2014. Sona geometry from Angola. Mathematics of an African tradition. ISTEG-University, Mozambique \& Lulu. (Mozambique). 248p.

12. GÓMEZ-LUNA, E.; FERNANDO-NAVAS, D; APONTEMAYOR, G.; BETANCOURT-BUITRAGO, L.A. 2014. Metodología para la revisión bibliográfica y la gestión de información de temas científicos, a través de su estructuración y sistematización. Dyna. (Colombia). 81(184):158-163.

13. HARRIS, P. 1984. Teaching about Time in Tribal Aboriginal Communities. Department of Education. (Darwin, Australia). 48p.

14. HOWSON, A.G. 1973. Developments in Mathematical Education. Proceedings of the Second lnternational Congress on Mathematical Education (Cambridge U. P., Cambridge). 330p.

15. HERNÁNDEZ, R.; FERNÁNDEZ, C.; BAPTISTA, P. 2014 Metodología de la investigación. McGraw-Hill (México) $600 \mathrm{p}$.

16. JONES, K.; TZEKAKI, M. 2016. Research on the teaching and learning of geometry. In: Gutiérrez, A.; Leder, G.; P. Boero, P. (Eds.). The Second Handbook of Research on the Psychology of Mathematics Education: The Journey Continues. Sende (Rotterdam). p.109-149.

17. LEAL, M. 2015. Mapping Time, Space and the Body. Indigenous Knowledge and Mathematical Thinking in Brazil. Sense Publishers (Rotterdam). 74p.

18. LEHMANN, C. 1989. Geometría Analítica. Edición 13 Editorial Limusa (México). 494p.

19. LEITHOLD, L. 1998. El cálculo. Séptima edición. Oxford University Press- Harla. (México). 1360p.

20. LEVINSON, S. 2003. Space in Language and cognition. Explorations in Cognitive Diversity. Cambridge University Press (New York). 389p.

21. LEWIS, D. 1976. Observations on route-finding and spatial orientation among the aboriginal peoples of the western desert region of central Australia. Oceania. (Australia). 46(4):249-282.

22. LLUIS, E. 1986. Enseñanza de geometría en América Latina. En: Morrys, E. (ed.). Enseñanza de geometría. Volumen 
5. Oficina Regional de Ciencia y Tecnología de la Unesco para América Latina y el Caribe - ROSTLAC. (Uruguay). 259p.

23. MINISTERIO DE INSTRUCCIÓN PÚBLICA. 1904. Decreto número 491 de 1904, por el cual se reglamenta la ley 89 de 1903, sobre instrucción pública. Bogotá, Colombia. 32p.

24. MINISTERIO DE EDUCACIÓN NACIONAL MINEDUCACIÓN. 1951. Decreto 0075 de 1951, por el cual se adopta el Plan de Estudios para la enseñanza secundaria y se dictan otras disposiciones. Bogotá, Colombia. $4 \mathrm{p}$.

25. MINISTERIO DE EDUCACIÓN NACIONAL MINEDUCACIÓN. 1951. Decreto 2550 de 1951, por el cual se introducen algunas modificaciones en el Plan de Estudios de Enseñanza Secundaria, y se deroga una disposición. Bogotá, Colombia. 3p.

26. MINISTERIO DE EDUCACIÓN NACIONAL MINEDUCACIÓN. 1962. Decreto 45 de 1962, por el cual se establece el Ciclo Básico de Educación Media, se determina el Plan de Estudios para el Bachillerato, y se fijan Calendario y Normas para evaluar el trabajo escolar. Bogotá, Colombia. 9p.

27. MINISTERIO DE EDUCACIÓN NACIONAL MINEDUCACIÓN. 1978. Decreto 1419 de 1978, por el cual se señalan las normas y orientaciones básicas para la administración curricular en los niveles de educación preescolar básica (primaria y secundaria) media vocacional e intermedia profesional. Bogotá, Colombia. 7p.

28. MINISTERIO DE EDUCACIÓN NACIONAL MINEDUCACIÓN. 1984. Decreto 1002 de 1984, por el cual se establece el Plan de Estudios Para la Educación Preescolar, Básica (Primaria y Secundaria) y Media Vocacional de la Educación Formal Colombiana. Bogotá, Colombia. 6p.

29. MINISTERIO DE EDUCACIÓN NACIONAL MINEDUCACIÓN. 2016. Derechos Básicos de Aprendizaje para Matemáticas. Volumen 2. Panamericana Formas E Impresos S.A. (Bogotá). 88p.

30. MINISTERIO DE EDUCACIÓN NACIONAL MINEDUCACIÓN. 2016. Documento Fundamentación Teórica de los Derechos Básicos de Aprendizaje (V2) y de las Mallas de Aprendizaje para el Área de Matemáticas. Universidad de Antioquia. (Medellín). 51p.

31. OWENS, K. 2015. Visuospatial Reasoning. An Ecocultural Perspective for Space, Geometry and Measurement Education. Springer. (Switzerland). 377p.
32. PATIÑO, G. 1977. Fundamentos de matemática superior moderna: cursos 5o. y 6o. de enseñanza media. Bedout. (Medellín). 504p.

33. PHILLIPS, H.B. 1948. Geometría analítica. Unión tipográfica editorial hispano-americana. (México). 227p.

34. PIAGET, J.; CHOQUET, G.; DIEUDONNNÉ, J.; THOM, R. 1978. La enseñanza de las matemáticas modernas. Alianza Editorial (Madrid). 401p.

35. PINXTEN, R.; VAN DOOREN, I.; HARVEY, F. 1983. The Anthropology of Space. University Pennsylvania Press. (Pennsylvania). 336p.

36. POSTIGO, L. 1964. Matemáticas. Editorial Ramón Sopena S.A. (España). 879p.

37. PRESIDENCIA DE LA REPÚBLICA DE COLOMBIA. 1974. Decreto 080 de 1974, por el cual se deroga el Decreto número 045 de 1962 y se dictan otras disposiciones sobre Educación Media. Bogotá, Colombia. 4p.

38. RIAÑO, C.A. 2017. Matemáticas 10. Guía del docente. Ministerio de Educación Nacional. (Colombia). 252p.

39. SANTALÓ, L. 1980. Situación de la enseñanza de la geometría frente a las nuevas tendencias de la Educación matemática. Rev. Bachillerato, suplemento. (España). 13:23-28.

40. SCHMIDT, M. 2006. Estándares Básicos de Competencias en Lenguaje, Matemáticas, Ciencias y Ciudadanas. Guía sobre lo que los estudiantes deben saber. Ministerio de Educación Nacional. Bogotá, Colombia. 183p.

41. STAKE, R.E. 1999. Investigación con estudio de casos. Segunda edición. Ediciones Morata, S.L. (Madrid). 159p.

42. TENBRINK, T.; KUHN, W. 2011. A model of spatial reference frames in language. In: Egenhofer, M.; Giudice, N.; Moratz, R.; Worboys, M. (eds.). Spatial Information Theory. Springer-Verlag. (Berlin). p.371-390.

43. TORRES-SÁNCHEZ, J.; SALAZAR HUR'TADO, L.A. 2002. Introducción a la historia de la ingeniería y de la educación en Colombia. Univ. Nacional de Colombia. 457p.

44. UNESCO. 1986. Estudios en Educación Matemática. Enseñanza de geometría. Volumen 5. En: Morrys, E. (ed.). Oficina Regional de Ciencia y Tecnología de la Unesco para América Latina y el Caribe - ROSTLAC. (Uruguay). 259p. 